\title{
Multiplicity distribution of electron-positron pairs created by strong external fields
}

\author{
Christoph Best and Walter Greiner \\ Institut für Theoretische Physik, Johann-Wolfgang-Goethe Universität, Postfach 111 932, D-6000 Frankfurt am Main, Germany \\ Gerhard Soff \\ Gesellschaft für Schwerionenforschung, Postfach 110 552, D-6100 Darmstadt, Germany
}

(Received 7 November 1991)

\begin{abstract}
We discuss the multiplicity distribution of electron-positron pairs created in the strong electromagnetic fields of ultrarelativistic heavy-ion transits. Based on nonperturbative expressions for the $N$-pair creation amplitudes, the Poisson distribution is derived by neglecting interference terms. The source of unitarity violation is identified in the vacuum-to-vacuum amplitude, and a perturbative expression for the mean number of pairs is given.
\end{abstract}

PACS number(s): $34.90 .+\mathrm{q}, 34.10 .+\mathrm{x}$

\section{INTRODUCTION}

While nearly all QED processes can be successfully computed in perturbation theory, it was recently observed by Baur [1] that pair creation in the electromagnetic fields of two ultrarelativistic heavy ions in an almost central collision explicitly violates unitarity in lowestorder perturbation theory, i.e., yields probabilities exceeding unity. This indicates that production of multiple pairs could be dominant over single-pair production. A similar effect was noted by Lippert et al. [2] in pair creation by Bremsstrahlung. It was demonstrated by Baur [3] using the sudden and quasiboson approximation that the resulting multiplicity distribution is a Poisson distribution, and that the average number of pairs in this approximation can be identified with the single-pair creation amplitude in second-order perturbation theory. A similar result was derived by Rhoades-Brown and Weneser [4] based on a resummation of the perturbation expansion.

We present here a general treatment of the pairproduction multiplicity in external-field problems based on the solutions of the Dirac equation in an external field. We first analyze the role of quantization using the pathintegral formulation of QED and derive an expression for the generating functional in terms of solutions of the Dirac equation. We then show that this generating functional is identical to the generating functional of a manyparticle theory of noninteracting fermions governed by the external-field Dirac equation. From this Dirac sea theory we derive expressions for the pair multiplicity and the $N$-pair creation probabilities in terms of $S$-matrix elements of the Dirac equation. We show that the source of the unitarity violation is not the perturbative solution of the Dirac equation, but the neglect of the vacuum-tovacuum amplitude which enters into the nonperturbative $\mathrm{N}$-pair amplitude as a multiplicative factor and is, in perturbation theory, assumed to be approximately unity. By neglecting interference terms the multiplicity distribution is then shown to be of Poisson form. An analysis of perturbation theory finally gives an expression for the (per- turbative) average multiplicity which coincides with the lowest-order perturbative single-pair creation probability.

\section{QUANTIZATION IN A TIME-DEPENDENT BASIS}

The explicit unitarity violation in second order requires either to sum higher-order contributions in perturbation theory or to seek for a nonperturbative treatment. The latter has become feasible with numerical coupledchannel computations of the Dirac equation [5]. Since pair creation is an effect of quantum-field theory we first investigate the role of field quantization in an externalfield problem in order to reduce the problem to the solution of the Dirac equation. While this has been accomplished before by employing operator calculus [6], we here present a treatment based on the path-integral formalism.

Both the classical equations of motion for a Dirac field as well as their quantization can be derived from the Lagrangian density $\mathcal{L}$ of the Dirac field $\psi$ in an external electromagnetic field $A_{\mu}$, which is given by

$$
\begin{aligned}
& \mathcal{L}\left(\psi, \partial_{\mu} \psi\right)=\mathcal{L}_{0}\left(\psi, \partial_{\mu} \psi\right)+\mathcal{L}_{\text {int }}\left(\psi, \partial_{\mu} \psi\right),
\end{aligned}
$$

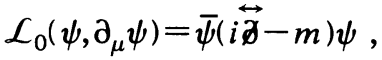

$$
\begin{aligned}
& \mathcal{L}_{\text {int }}\left(\psi, \partial_{\mu} \psi\right)=-e \bar{\psi} \gamma^{\mu} \psi A_{\mu} \text {. }
\end{aligned}
$$

The symbol $\overleftrightarrow{g}$ indicates the symmetric derivative

$$
\overleftrightarrow{\not}=\frac{1}{2} \vec{\not}-\frac{1}{2} \overleftarrow{\not}
$$

Variation of the action and the resulting Euler-Lagrange equations

$$
\frac{\partial \mathcal{L}}{\partial \psi}-\partial_{\mu} \frac{\partial \mathcal{L}}{\partial\left(\partial_{\mu} \psi\right)}=0
$$

lead to the classical equation of motion

$$
\begin{aligned}
& (i \not-e A-m) \psi=0, \\
& \bar{\psi}(i \overleftarrow{\partial}+e A+m)=0,
\end{aligned}
$$


which is the Dirac equation and its conjugate.

The path integral offers a suitable mathematical tool to discuss differences between a classical and a quantized theory (i.e., quantum corrections) since the classical motion plays a central role in it. In the path-integral formalism the two-point function $G\left(x, x^{\prime}\right)$, which is the vacuum expectation value of the time-ordered field operators, is computed by a path integral according to

$$
\begin{aligned}
G\left(x, x^{\prime}\right) & =\left\langle 0\left|T \hat{\psi}(x) \widehat{\bar{\psi}}\left(x^{\prime}\right)\right| 0\right\rangle \\
& =\int \mathcal{D} \bar{\psi} \mathcal{D} \psi \psi(x) \bar{\psi}\left(x^{\prime}\right) e^{i S[\psi, \bar{\psi}]} .
\end{aligned}
$$

This is a functional integral over all classical paths that the field can take, weighed with a complex measure given by the action functional

$$
S[\psi]=\int d^{4} x \mathcal{L}\left(\psi(x), \partial_{\mu} \psi(x)\right) .
$$

The propagator can be expressed as a functional derivative of the normalized generating functional

$$
\bar{W}[\eta, \bar{\eta}]=\frac{W[\eta, \bar{\eta}]}{W[0]}
$$

which originates from the unnormalized generating functional

$$
W[\eta, \bar{\eta}]=\int \mathscr{D} \bar{\psi} \mathcal{D} \psi \exp \left[i S[\psi, \bar{\psi}]+i \int d^{4} x[\bar{\eta}(x) \psi(x)+\bar{\psi}(x) \eta(x)]\right]
$$

The external currents $\eta(x), \bar{\eta}(x)$ serve as dummy variables; the normalization is chosen in such a way that the generating functional without external currents is unity. Since the field is fermionic, the classical fields $\psi$ are to be considered anticommuting Grassmann variables, and the appropriate formulas for executing the Gaussian integration must be used. All results of perturbation theory can be derived from path integrals in a similar way as they can be deduced from operator quantization, but, as we will see, the path integral allows one to utilize the classical solutions of the field equations as a basis.

In particular it is possible to use the set of classical solutions of the equations of motion (for different initial conditions) as a basis. Let therefore $\left\{\psi_{n}(x)\right\}$ be a timedependent, complete, at all times orthonormal set of solutions of the Dirac equation in a given time-dependent external field $A_{\mu}$, i.e., for every $n$

$$
(i ð-e A-m) \psi_{n}=0
$$

holds, and at all times $t$

$$
\int d^{3} x \bar{\psi}_{n}(\mathbf{x}, t) \psi_{n^{\prime}}(\mathbf{x}, t)=\delta_{n n^{\prime}}
$$

for all $n, n^{\prime}$ (in a box normalization this can also be used for continuum states). Since time evolution is unitary, these two equations do not contradict each other. The integration variable $\psi$ of the path integral can now be expanded in this basis,

$$
\psi(\mathbf{x}, t)=\sum_{n} a_{n}(t) \psi_{n}(\mathbf{x}, t) .
$$

The amplitudes $a_{n}(t)$ can be substituted for the field variables $\psi(x)$ in the path integral; in order that the $\psi(\mathbf{x}, t)$ resulting from (13) are Grassmann numbers, the $a_{n}$ must anticommute. The Lagrangian density $\mathcal{L}_{0}$ of the free field is now after substituting (13),

$$
\begin{aligned}
\mathcal{L}_{0}=\sum_{n, n^{\prime}}\{ & a_{n}^{*}(t)\left[\bar{\psi}_{n}(i \overleftrightarrow{\not}-m) \psi_{n^{\prime}}\right] a_{n^{\prime}}(t) \\
& \left.+i\left[a_{n}^{*}(t) \overleftrightarrow{\partial}_{t} a_{n^{\prime}}(t)\right]\left(\bar{\psi}_{n} \gamma^{0} \psi_{n^{\prime}}\right)\right\} .
\end{aligned}
$$

The differential operator can be rewritten according to its definition as

$$
(i \overleftrightarrow{\not \partial}-m)=\frac{1}{2}(i \not \partial-m)-\frac{1}{2}(i \overleftarrow{\not}+m)
$$

Since the basis functions $\psi_{n}$ satisfy

$$
\begin{aligned}
& (i \not-m) \psi_{n}=e A \psi_{n}, \\
& \bar{\psi}_{n}(i \overleftarrow{\partial}+m)=-e \bar{\psi}_{n} A,
\end{aligned}
$$

the Lagrangian density in terms of the $a_{n}$ reads

$$
\begin{aligned}
\mathcal{L}_{0=} \sum_{n, n^{\prime}}\left\{a_{n}^{*}(t) e \bar{\psi}_{n} A \psi_{n^{\prime}} a_{n^{\prime}}(t)\right. \\
\left.\quad+i\left[a_{n}^{*}(t) \overleftrightarrow{\mathrm{\partial}}_{t} a_{n^{\prime}}(t)\right]\left(\bar{\psi}_{n} \gamma^{0} \psi_{n^{\prime}}\right)\right\} .
\end{aligned}
$$

The interaction contribution to the Lagrangian density is

$$
\mathcal{L}_{\text {int }}=-e \sum_{n, n^{\prime}} a_{n}^{*}(t) \bar{\psi}_{n} A \psi_{n^{\prime}} a_{n^{\prime}}(t)
$$

and the total Lagrangian density therefore becomes

$$
\mathcal{L}=\sum_{n, n^{\prime}} i\left[a_{n}^{*}(t) \overleftrightarrow{\mathrm{\partial}}_{t} a_{n^{\prime}}(t)\right]\left(\bar{\psi}_{n} \gamma^{0} \psi_{n^{\prime}}\right)
$$

Due to the orthogonality properties of the basis functions the Lagrange function takes the following simple form after integration over spatial coordinates:

$L(t)=\int d^{3} x \mathcal{L}\left(\psi(x), \partial_{\mu} \psi(x)\right)=\sum_{n} i a_{n}^{*}(t) \overleftrightarrow{\partial}_{t} a_{n}(t)$

The Lagrange function describes the behavior of the dynamical system constituted by the $a_{n}(t)$. By variation of the action the classical equations of motion are

$$
\frac{\partial L}{\partial a_{n}}-\frac{\partial}{\partial t} \frac{\partial L}{\partial \dot{a}_{n}}=0 \rightarrow i \dot{a}_{n}(t)=0 \text {. }
$$

The amplitudes $a_{n}(t)$ are therefore constant in time; this is what we expect since the basis functions are full solutions of the equation of motion (unlike the coupledchannel formalism in which the basis functions satisfy only a part of the equations of motion). Quantum corrections to these equations manifest themselves by a spontaneous (not externally induced) leap of the system from 
one classical solution into another ("tunneling").

Since the transformation of field variables to amplitudes $a_{n}$ is [due to the normalization (12)] unitary, the basis transformation

$$
\mathscr{D} \psi \mathscr{D} \bar{\psi}=\mathscr{D} a_{1} \cdots \mathcal{D} a_{n} \mathscr{D} a_{1}^{*} \cdots \mathcal{D} a_{n}^{*}
$$

can be performed in the path integral and the generating functional can be cast in the following simple form:

$$
\begin{array}{r}
W[\eta, \bar{\eta}]=\int \mathcal{D} a_{1} \cdots \mathcal{D} a_{n} \cdots \mathcal{D} a_{1}^{*} \cdots \mathcal{D} a_{n}^{*} \cdots \exp \left\{i \sum _ { n } \left[\int d t \int d t^{\prime} a_{n}^{*}\left(t^{\prime}\right) B\left(t^{\prime}, t\right) a_{n}(t)\right.\right. \\
\left.\left.+\int d t\left[\eta_{n}^{*}(t) a_{n}(t)+a_{n}^{*}(t) \eta_{n}(t)\right]\right]\right\},
\end{array}
$$

where the integral kernel is

$$
B\left(t^{\prime}, t\right)=i \overleftrightarrow{\partial}_{t} \delta\left(t^{\prime}-t\right)
$$

The quantities $\eta_{n}(t)$ replace the current $\eta(x)$ according to

$$
\eta_{n}(t)=\int d^{3} x \bar{\psi}_{n}(\mathbf{x}, t) \eta(\mathbf{x}, t)
$$

We assume that the following formula for complex Gaussian path integrals over Grassmann variables holds [7]:

$$
\begin{aligned}
\int \mathscr{D} a^{*} \mathscr{D} a \exp \left\{i \int d t^{\prime} \int d t a^{*}\left(t^{\prime}\right) B\left(t^{\prime}, t\right) a(t)+i \int d t\left[\eta^{*}(t) a(t)+a^{*}(t) \eta(t)\right]\right\} \\
=\mathcal{N} \exp \left\{-i \int d t^{\prime} \int d t \eta^{*}\left(t^{\prime}\right) B^{-1}\left(t^{\prime}, t\right) \eta(t)\right\} .
\end{aligned}
$$

The symbol $\mathcal{N}$ stands for an arbitrary, not necessarily the same in every equation, constant factor. With this formula the path integral can be performed and reduces to

$$
W[\eta, \bar{\eta}]=\mathcal{N} \exp \left\{-i \sum_{n} \int d t \int d t^{\prime} \eta_{n}^{*}\left(t^{\prime}\right) B^{-1}\left(t^{\prime}, t\right) \eta_{n}(t)\right\} .
$$

The transition to the normalized generating functional is simply accomplished by dropping the constant factor $\mathcal{N}$. The inverse of the operator kernel $B\left(t^{\prime}, t\right)$ can be found by a Fourier transform; it is

$$
B\left(\omega^{\prime}, \omega\right)=-\omega(2 \pi) \delta\left(\omega-\omega^{\prime}\right)
$$

and therefore

$$
\begin{aligned}
B^{-1}\left(t^{\prime}, t\right) & =(2 \pi)^{-2} \int d \omega^{\prime} d \omega B^{-1}\left(\omega^{\prime}, \omega\right) e^{i\left(\omega t-\omega^{\prime} t^{\prime}\right)} \\
& =-(2 \pi)^{-1} \int d \omega \frac{1}{\omega} e^{i \omega\left(t-t^{\prime}\right)}
\end{aligned}
$$

This integral is a representation of the step function $\Theta\left(t-t^{\prime}\right)$. Depending on how the integration contour around the singularity at $\omega=0$ in the integrand is chosen, its value is

$$
\begin{aligned}
B^{-1}\left(t^{\prime}, t\right) & =-(2 \pi)^{-1} \int d \omega \frac{1}{\omega \pm i \epsilon} e^{i \omega\left(t-t^{\prime}\right)} \\
& = \begin{cases}-i \Theta\left(t^{\prime}-t\right) & \text { positive sign } \\
i \Theta\left(t-t^{\prime}\right) & \text { negative sign }\end{cases}
\end{aligned}
$$

This choice fixes the boundary conditions of the propagator derived from the generating functional; it can be made separately for each state and determines whether $\psi_{n}$ describes an electron or a positron. The normalized generating functional finally becomes

$$
\bar{W}[\eta, \bar{\eta}]=\exp \left\{-\sum_{n}\left[\int d t \int d t^{\prime} \eta_{n}^{*}\left(t^{\prime}\right) S_{n}\left(t^{\prime}-t\right) \eta_{n}(t)\right)\right\},
$$

where the one-dimensional propagator $S_{n}\left(t^{\prime}-t\right)$ takes one of the following two forms depending on whether $n$ lies above or below the Fermi level $F$, i.e., the highest state occupied in the Dirac vacuum:

$$
S_{n}\left(t^{\prime}-t\right)= \begin{cases}\Theta\left(t^{\prime}-t\right), \quad n>F \\ -\Theta\left(t-t^{\prime}\right), \quad n<F\end{cases}
$$

The propagator (i.e., the 2-point function) is now found as a functional derivative of the generating functional

$$
S\left(x^{\prime}, x\right)=\frac{1}{i^{2}} \frac{\delta^{2} \bar{W}}{\delta \bar{\eta}\left(x^{\prime}\right) \delta \eta(x)} .
$$

It is

$$
\begin{aligned}
& \frac{\delta}{\delta \eta(x)}=\sum_{n} \bar{\psi}_{n}(\mathbf{x}, t) \frac{\delta}{\delta \eta_{n}(t)}, \\
& \frac{\delta}{\delta \bar{\eta}(x)}=\sum_{n} \psi_{n}(\mathbf{x}, t) \frac{\delta}{\delta \eta_{n}^{*}(t)},
\end{aligned}
$$


and therefore

$$
\begin{aligned}
S\left(x^{\prime}, x\right) & =\sum_{n, n^{\prime}} \psi_{n^{\prime}}\left(\mathbf{x}^{\prime}, t^{\prime}\right) \frac{\delta^{2} W}{i \delta \eta_{n^{\prime}}^{*}\left(t^{\prime}\right) i \delta \eta_{n}(t)} \bar{\psi}_{n}(\mathbf{x}, t) \\
& =\sum_{n} \psi_{n}\left(\mathbf{x}^{\prime}, t^{\prime}\right) S_{n}\left(t^{\prime}-t\right) \bar{\psi}_{n}(\mathbf{x}, t)
\end{aligned}
$$

This expression for the propagator is identical to the representation of the Feynman propagator in an external field by the solutions of the Dirac equation. Therefore, path-integral quantization has not changed the propagator; this was not to be expected since the Lagrangian density of the Dirac equation in an external field is still quadratic in the Dirac fields. The use of the Dirac equation in an external field is thus justified. It can be shown that the true quantum effects in perturbation theory can be identified with those Feynman diagrams that contain loops while the tree diagrams constitute the limit $\hbar \rightarrow 0$ $[8]$.

\section{FORMALISM}

\section{A. Many-particle theory and Fock space}

Pair creation is a process that cannot be described in the framework of a single-particle theory. In canonical quantization the many-particle interpretation arises naturally since the canonical field operators satisfy just those commutation relations that generate the (anti-)symmetric subspace in the space of many-particle wave functions. In path-integral quantization we can show that the generating functional derived above coincides with the generating functional of a system of many noninteracting particles, each of which obeys the Dirac equation.

As in canonical quantization, antiparticles are introduced by redefining a negative-energy electron destruction operator to be a new positron creation operator, and accordingly for negative-energy electron creation operators. The Dirac vacuum is annihilated by both electron and positron destruction operators, which can be implemented formally by filling all negative-energy electron states of the Dirac vacuum. As long as the particles do not interact with each other, this picture is consistent, since-by virtue of the redefinition of creation and destruction operators - the Dirac sea does not influence measurable quantities. If interactions between particles are considered, the normal-ordering procedure excludes Dirac sea electrons from being measured, so that only holes in the Dirac sea, i.e., positrons, are physical.

We write the external-field Dirac equation in Hamiltonian form

$$
i \frac{\partial}{\partial t}|\psi\rangle=\hat{H}|\psi\rangle
$$

and decompose the Hamilton operator $\hat{H}(t)=\hat{H}_{0}$ $+\widehat{H}_{\text {int }}(t)$ into an unperturbed time-independent part $\hat{H}_{0}$ and a time-dependent interaction part $\hat{H}_{\text {int }}$ which vanishes for $t \rightarrow \pm \infty$. If $\{|n\rangle\}$ is the set of eigenstates of $\hat{H}_{0}$

$$
\hat{H}_{0}|n\rangle=E_{n}|n\rangle \text {, }
$$

then channel amplitudes can be introduced by

$$
a(n)=\langle n \mid \psi\rangle e^{i E_{n} t}
$$

which satisfy the coupled-channel equations

$$
i \frac{\partial a(n)}{\partial t}=\sum_{m} V_{n m}(t) a(m)
$$

with the time-dependent channel couplings

$$
V_{n m}(t)=\left\langle n\left|\bar{H}_{\text {int }}\right| m\right\rangle e^{i\left(E_{n}-E_{m}\right) t},
$$

which vanish at $t \rightarrow \pm \infty$. Accordingly, the wave function of a system consisting of $N$ particles is

$$
a\left(n_{1}, \ldots, n_{N}\right) ;
$$

it gives the amplitude that particle 1 is in state $n_{1}$, particle 2 in $n_{2}$, and so on. Since the particles are identical, we can introduce a Fock space spanned by the algebra of creation and destruction operators $\widehat{a}_{n}, \widehat{a}_{n}^{\dagger}$, satisfying anticommutation relations

$$
\left\{\widehat{a}_{n}, \widehat{a}_{m}^{\dagger}\right\}=\widehat{a}_{n} \widehat{a}_{m}^{\dagger}+\widehat{a}_{m}^{\dagger} \widehat{a}_{n}=\delta_{n m}
$$

and a basis given by the occupation number representation

$$
\left|k_{1}, k_{2}, \ldots\right\rangle
$$

whose quantum numbers $k_{n}$ specify how many particles are in the single-particle state $n$; the action of creation and destruction operators is then

$$
\begin{aligned}
& \widehat{a}_{n}^{\dagger}\left|k_{1}, k_{2}, \ldots\right\rangle=\sqrt{k_{n}+1}\left|k_{1}, \ldots, k_{n}+1, \ldots\right\rangle, \\
& \hat{a}_{n}\left|k_{1}, k_{2}, \ldots\right\rangle=\sqrt{k_{n}}\left|k_{1}, \ldots, k_{n}-1, \ldots\right\rangle .
\end{aligned}
$$

The state in which no particles are present is designated by $|0\rangle$; it satisfies

$$
\widehat{a}_{n}|0\rangle=0 \text {. }
$$

The relationship of a many-particle wave function and the corresponding Fock space vector is given by the following relations:

$$
\begin{aligned}
& |\Psi\rangle=\frac{1}{N !} \sum_{n_{1}} \cdots \sum_{n_{N}} a\left(n_{1}, \ldots, n_{N}\right) \widehat{a}_{n_{1}}^{\dagger} \cdots \widehat{a}_{n_{N}}^{\dagger}|0\rangle, \\
& a\left(n_{1}, \ldots, n_{N}\right)=\left\langle 0\left|\widehat{a}_{n_{N}} \cdots \widehat{a}_{n_{1}}\right| \Psi\right\rangle .
\end{aligned}
$$

The factor $1 / N$ ! accounts for the fact that the sum runs over all $N$ ! permutations of the $n_{i}$ that designate the same Hilbert space vector. The dynamics of $|\Psi\rangle$ is governed by

$$
i \frac{\partial|\Psi\rangle}{\partial t}=\hat{H}_{\text {Fock }}|\Psi\rangle
$$

with the Hamiltonian

$$
\widehat{H}_{\mathrm{Fock}}=\sum_{n, n^{\prime}} V_{n n^{\prime}} \widehat{a}_{n}^{\dagger} \widehat{a}_{n^{\prime}}
$$

Antiparticle operators are introduced in the framework of Fock space by defining 


$$
\widehat{a}_{n}= \begin{cases}\hat{b}_{n}, & n>0 \\ \hat{d}_{n}^{\dagger}, & n<0\end{cases}
$$

where $n>0$ is to be read symbolically that the energy of the state $n$ is positive (or, more general, lies above the Fermi level). The operators $b_{n}, d_{n}$ can be interpreted as destruction operators for electrons and positrons, respectively. The Dirac vacuum is then the state which is annihilated by all (electron and positron) destruction operators, i.e., it satisfies

$$
\hat{b}_{p}|\overline{0}\rangle=\hat{d}_{q}|\overline{0}\rangle=0 \text {. }
$$

The particle and antiparticle operators have the same commutation relations as the $\hat{a}_{n}$. To derive measurable quantities like charge density, or to include interactions with an electromagnetic field, the normal-ordering procedure accounts for the fact that only positive-energy electrons and positrons, but not negative-energy Dirac sea electrons, are physical.

\section{B. Computation of many-particle amplitudes from single-particle theory}

If the particles of the Dirac sea do not interact with each other, then the many-particle state vector can be computed from single-particle wave functions by factorization and appropriate symmetrization: With the singleparticle amplitudes given as $a_{i}(n)$, where $i$ designates a particle and $n$ a state, the (anti-)symmetrical manyparticle state is

$$
\begin{array}{r}
|\Psi\rangle=\frac{1}{N !} \sum_{n_{1}} \cdots \sum_{n_{N}} a_{1}\left(n_{1}\right) \ldots a_{N}\left(n_{N}\right) \\
\quad \times \hat{a}^{\dagger}\left(n_{1}\right) \ldots \hat{a}^{\dagger}\left(n_{N}\right)|\overline{0}\rangle .
\end{array}
$$

A system whose asymptotical initial state is the Dirac vacuum consists of as many particles as the Dirac sea contains, and the single-particle amplitudes for $t \rightarrow-\infty$ are

$$
a_{q}(n)=\delta_{n q}
$$

where the index $i$ is now replaced by an antiparticle index $q<0$. To solve then the many-particle problem of the Dirac sea, knowledge of the time development of any Dirac sea state is required.

This knowledge can be expressed by introducing Heisenberg operators into the formalism. Applying a time-dependent Heisenberg operator $\hat{a}_{n}^{\dagger}(t)$ to the vacuum state just creates that state which would have resulted from time development of the asymptotic state $\hat{a}_{n}^{\dagger}|0\rangle$. Heisenberg and Schrödinger operators are related by a unitary transformation whose coefficients are the singleparticle amplitudes

$$
\begin{aligned}
& \hat{\widetilde{a}}_{p}^{\dagger}(t)=\sum_{n} a_{p n}(t) \hat{a}_{n}^{\dagger}, \\
& \hat{a}_{n}^{\dagger}=\sum_{p} a_{p n}^{*}(t) \hat{\widetilde{a}}_{p}^{\dagger}(t) .
\end{aligned}
$$

The state at a time $t$ is therefore

$$
\left|\Psi_{t}\right\rangle=\frac{1}{N !} \hat{\widetilde{a}}_{p_{1}}^{\dagger}(t) \cdots \hat{\widetilde{a}}_{p_{N}}^{\dagger}(t)|\overline{0}\rangle,
$$

where the state $p_{i}$ of the $i$ th particle at $t \rightarrow-\infty$ now enters explicitly. Since the transformation is unitary the Heisenberg operators satisfy the same commutation relations as the Schrödinger operators, i.e., for fermions

$$
\left\{\hat{\widetilde{a}}_{n}(t), \hat{\widehat{a}}_{m}^{\dagger}(t)\right\}=\delta_{n m} \text {. }
$$

Especially the relations

$$
\hat{\widehat{a}}_{n}^{\dagger}(t)\left|\Psi_{t}\right\rangle=0, \text { if } n \in\left\{p_{1}, \ldots, p_{N}\right\}
$$

and

$$
\widehat{\widehat{a}}_{n}(t)\left|\Psi_{t}\right\rangle=0 \text { if } n \notin\left\{p_{1}, \ldots, p_{N}\right\}
$$

hold, and the Heisenberg number operator is

$$
\begin{aligned}
\left\langle\Psi\left|\hat{\widehat{N}}_{n}\right| \Psi\right\rangle & =\left\langle\Psi\left|\hat{\widetilde{a}}_{n}^{\dagger} \hat{\widetilde{a}}_{n}\right| \Psi\right\rangle \\
& = \begin{cases}1 & \text { if } n \in\left\{p_{1}, \ldots, p_{N}\right\} \\
0 & \text { otherwise } .\end{cases}
\end{aligned}
$$

The generating functional of this many-particle theory is defined in terms of the Heisenberg operators as

$$
W[\widetilde{\eta}, \overline{\tilde{\eta}}]=\left\langle 0\left|T \exp \left\{i \int d t \sum_{p}\left[\widetilde{\eta}_{p}^{*}(t) \widehat{\widetilde{a}}_{p}(t)+\widetilde{\eta}_{p}(t) \hat{\widetilde{a}}_{p}^{\dagger}(t)\right]\right\}\right| 0\right\rangle,
$$

where the currents $\widetilde{\eta}, \overline{\widetilde{\eta}}$ are dummy variables to extract $n$-point functions. Substituting (54) and introducing new currents [which we later will identify with the currents $(25)$ ]

$$
\eta_{n}(t)=\sum_{p} \widetilde{\eta}_{p}(t) a_{p n}(t)
$$

this can be rewritten as

$$
W[\eta, \bar{\eta}]=\left\langle 0\left|T \exp \left\{i \int d t \sum_{n}\left[\eta_{n}^{*}(t) \widehat{a}_{n}(t)+\eta_{n}(t) \widehat{a}_{n}^{\dagger}(t)\right]\right\}\right| 0\right\rangle .
$$

Though the Schrödinger operators are time independent, we must keep the time argument to perform the time ordering 
correctly. Wick's theorem [8] enables us to rewrite the last expression as

$$
\begin{aligned}
W[\eta, \bar{\eta}]= & \left\langle 0\left|: \exp \left\{i \int d t \sum_{n}\left[\eta_{n}^{*}(t) \widehat{a}_{n}+\eta_{n}(t) \hat{a}_{n}^{\dagger}\right]\right\}:\right| 0\right\rangle \\
& \times \exp \left\{-\iint d t d t^{\prime} \sum_{n, n^{\prime}} \eta_{n^{\prime}}^{*}\left(t^{\prime}\right)\left\langle 0\left|T \widehat{a}_{n^{\prime}}\left(t^{\prime}\right) \hat{a}_{n}^{\dagger}(t)\right| 0\right\rangle \eta_{n}(t)\right\} .
\end{aligned}
$$

The normal-ordered term on the right-hand side drops out, and the time-ordered product is

$$
\left\langle 0\left|T \widehat{a}_{n^{\prime}}\left(t^{\prime}\right) \widehat{a}_{n}^{\dagger}(t)\right| 0\right\rangle=\delta_{n n^{\prime}} \Theta\left(t^{\prime}-t\right) .
$$

We thus find

$$
W[\eta, \bar{\eta}]=\exp \left\{-\sum_{n}\left[\int d t \int d t^{\prime} \eta_{n}^{*}\left(t^{\prime}\right) \Theta\left(t^{\prime}-t\right) \eta_{n}(t)\right)\right\},
$$

which is identical to expression (31) when the forward propagator is chosen. This is correct since we have not yet made use of antiparticles.

The preceding relations can be utilized to reduce any matrix element of the Schrödinger operators to an expression in terms of the single-particle amplitudes. The number operator, e.g., for a particle in state $n$ is according to (54)

$$
\widehat{N}_{n}=\hat{b}_{n}^{\dagger} \hat{b}_{n}=\hat{a}_{n}^{\dagger} \widehat{a}_{n}=\sum_{p q} a_{p n}^{*}(t) a_{q n}(t) \hat{\widetilde{a}}_{p}^{\dagger} \hat{\widetilde{a}}_{q},
$$

and therefore its expectation value at a time $t$ reads

$$
\begin{aligned}
\left\langle\Psi_{t}\left|\hat{N}_{n}\right| \Psi_{t}\right\rangle & =\sum_{p}\left|a_{p n}(t)\right|^{2}\left\langle\Psi_{t}\left|\hat{\hat{N}}_{p}\right| \Psi_{t}\right\rangle \\
& =\sum_{p<0}\left|a_{p n}(t)\right|^{2} .
\end{aligned}
$$

This is the well-known result [6]. For antiparticles $n<0$ it is

$$
\widehat{N}_{n}=\widehat{d}_{n}^{\dagger} \widehat{d}_{n}=\widehat{a}_{n} \widehat{a}_{n}^{\dagger}=1-\widehat{a}_{n}^{\dagger} \widehat{a}_{n}
$$

and its expectation value accordingly

$$
\left\langle\Psi_{t}\left|\hat{N}_{n}\right| \Psi_{t}\right\rangle=1-\sum_{p<0}\left|a_{p n}(t)\right|^{2}
$$

If the theory contains antiparticles, creation and destruction operators in the Heisenberg picture have to be introduced corresponding to those in the Schrödinger picture,

$$
\begin{aligned}
& \hat{b}_{p}(t)=\sum_{n<0} a_{p n}^{*}(t) \hat{d}_{n}^{\dagger}+\sum_{n>0} a_{p n}^{*}(t) \hat{b}_{n}, \\
& \hat{d}_{q}(t)=\sum_{n<0} a_{q n}(t) \hat{d}_{n}+\sum_{n>0} a_{q n}(t) \hat{b}_{n}^{\dagger}
\end{aligned}
$$

and

$$
\begin{aligned}
& \hat{b}_{p}=\sum_{n<0} a_{n p}(t) \hat{\vec{d}}_{n}^{\dagger}(t)+\sum_{n>0} a_{n p}(t) \hat{\vec{b}}_{n}(t), \\
& \hat{d}_{q}=\sum_{n<0} a_{n q}^{*}(t) \hat{d}_{n}(t)+\sum_{n>0} a_{n q}^{*}(t) \hat{\widehat{b}}_{n}^{\dagger}(t) .
\end{aligned}
$$

From these expressions pair creation amplitudes can be computed. Using the fact that $\widehat{\vec{b}}_{n}$ and $\hat{\vec{d}}_{n}$ annihilate the vacuum, the amplitude for the presence of exactly one pair $(p, q)$ at a given time then is

$$
\begin{aligned}
S_{p q}= & \left\langle\overline{0}\left|\hat{d}_{q} \hat{b}_{p}\right| \Psi\right\rangle \\
= & \sum_{n<0} \sum_{n^{\prime}<0} a_{n q}^{*} a_{n^{\prime} p}\left\langle\overline{0}\left|\hat{\vec{d}}_{n} \hat{\widetilde{d}}_{n^{\prime}}^{\dagger}\right| \Psi\right\rangle \\
& +\sum_{n>0} \sum_{n^{\prime}<0} a_{n q}^{*} a_{n^{\prime} p}\left\langle\overline{0}\left|\hat{\vec{b}}_{n}^{\dagger} \hat{\widetilde{d}}_{n^{\prime}}^{\dagger}\right| \Psi\right\rangle .
\end{aligned}
$$

By virtue of the commutation relations, this is

$S_{p q}=\sum_{n<0} a_{n q}^{*} a_{n p}\langle\overline{0} \mid \Psi\rangle+\sum_{n>0} \sum_{n^{\prime}<0} a_{n q}^{*} a_{n^{\prime} p}\left\langle\overline{0}\left|\hat{\vec{b}}_{n}^{\dagger} \hat{\widetilde{d}}_{n^{\prime}}^{\dagger}\right| \Psi\right\rangle$.

The second term cannot be further simplified but it can be argued that its contribution is small. The vacuum expectation value $\left\langle\overline{0}\left|\hat{\vec{b}}_{n}^{\dagger} \hat{\vec{d}}_{n}^{\dagger}\right| \Psi\right\rangle$ represents the amplitude that a state containing initially a pair $\left(n, n^{\prime}\right)$ becomes the vacuum, i.e., an annihilation amplitude. This is to be compared to the vacuum-to-vacuum amplitude $\langle\overline{0} \mid \Psi\rangle$ and will therefore be of higher order. Furthermore, the coefficients $a_{n q}^{*} a_{n^{\prime} p}$ in front of the second term both fall into the nondiagonal quadrants of the single-particle scattering matrix while one of the coefficients $a_{n q}^{*} a_{n p}$ in front of the other term is in the diagonal quadrant. In fact, the perturbative expansion of the coefficients in the first term will be

$$
\sum_{n<0} a_{n q}^{*} a_{n p} \approx \sum_{n<0}\left(\delta_{n q}+a_{n q}^{(1) *}\right) a_{n p}^{(1)} \approx a_{q p}^{(1)}
$$

and is therefore of first order while the coefficient in the second term will be of second order.

The factor $\langle\overline{0} \mid \Psi\rangle$ is the amplitude that the vacuum remains unchanged and is therefore not accessible to perturbation theory where it is assumed to be near to unity.

\section{RESULTS}

\section{A. The multiplicity distribution}

Amplitudes for the creation of several pairs can be computed similarly. It is useful to introduce reduced one-pair amplitudes which do not contain the vacuumto-vacuum amplitude

$$
\bar{S}_{p q}=\frac{S_{p q}}{\langle\overline{0} \mid \Psi\rangle}=\sum_{n<0} a_{n q}^{*}(t) a_{n p}(t) .
$$

With this definition and the above approximation the 
two-pair amplitude becomes

$$
S_{p q p^{\prime} q^{\prime}}=\langle\overline{0} \mid \Psi\rangle\left(\bar{S}_{p q} \bar{S}_{p^{\prime} q^{\prime}}-\bar{S}_{p q^{\prime}} \bar{S}_{p^{\prime} q}\right) \text {. }
$$

The two terms correspond to the two possible exchange graphs in Feynman perturbation theory. The probability for the production of two pairs with arbitrary energies is then the sum over the absolute squares of the amplitudes,

$$
\begin{gathered}
P_{2}=\left[\frac{1}{2 !}\right]^{2} \sum_{p>0} \sum_{q<0} \sum_{p^{\prime}>0} \sum_{q^{\prime}<0}|\langle\overline{0} \mid \Psi\rangle|^{2}\left[\left|\bar{S}_{p q}\right|^{2}\left|\bar{S}_{p^{\prime} q^{\prime}}\right|^{2}\right. \\
\left.+\left|\bar{S}_{p q^{\prime}}\right|^{2}\left|\bar{S}_{p^{\prime} q}\right|^{2}-2 \operatorname{Re}\left(\bar{S}_{p q}^{*} \bar{S}_{p^{\prime} q^{\prime}}^{*} \bar{S}_{p q^{\prime}} \bar{S}_{p^{\prime} q}\right)\right]
\end{gathered}
$$

The factor $(1 / 2 !)^{2}$ accounts for the double summation over identical states in $\left(p p^{\prime}\right)$ and $\left(q q^{\prime}\right)$. The first two terms inside the summation yield just the one-pair probability $P_{1}$ whereas the third term is an interference term which cannot be reduced further,

$$
\begin{aligned}
P_{1}= & \sum_{p>0} \sum_{q<0}|\langle\overline{0} \mid \Psi\rangle|^{2}\left|\bar{S}_{p q}\right|^{2}=|\langle\overline{0} \mid \Psi\rangle|^{2} \bar{P}_{1}, \\
P_{2}= & \frac{1}{2 !}|\langle\overline{0} \mid \Psi\rangle|^{2} \bar{P}_{1}^{2}-\frac{1}{2 !}|\langle\overline{0} \mid \Psi\rangle|^{2} \\
& \times \operatorname{Re} \sum_{p>0} \sum_{q<0} \sum_{p^{\prime}>0} \sum_{q^{\prime}<0}\left(\bar{S}_{p q}^{*} \bar{S}_{p^{\prime} q^{\prime}}^{*} \bar{S}_{p q^{\prime}} \bar{S}_{p^{\prime} q}\right),
\end{aligned}
$$

where the reduced single-pair creation probability

$$
\bar{P}_{1}=\sum_{p>0} \sum_{q<0}\left|\bar{S}_{p q}\right|^{2}=\frac{P_{1}}{|\langle\overline{0} \mid \Psi\rangle|^{2}}
$$

has been introduced. The summation in this last term, e.g., can be grouped in this way,

$$
\begin{aligned}
\sum_{p>0} \sum_{p^{\prime}>0}\left[\left[\sum_{q<0} \bar{S}_{p q}^{*} \bar{S}_{p^{\prime} q}\right]\right. & {\left.\left[\sum_{q^{\prime}<0} \bar{S}_{p^{\prime} q^{\prime}}^{*} \bar{S}_{p q^{\prime}}\right]\right] } \\
& =\sum_{p>0} \sum_{p^{\prime}>0}\left|\sum_{q<0} \bar{S}_{p q}^{*} \bar{S}_{p^{\prime} q}\right|^{2} .
\end{aligned}
$$

The innermost summation contains the $S$-matrix elements that link $p$ and $p^{\prime}$ to $q$, the state over which is summed. If the phase of these $S$-matrix elements varies sufficiently fast in the course of the $q$ summation, the terms cancel out and can be neglected compared to the other terms which do not depend on the phase.

In this approximation the integrated two-pair probability results as

$$
P_{2}=|\langle\overline{0} \mid \Psi\rangle|^{2} \frac{1}{2 !} \bar{P}_{1}^{2} .
$$

One can find similarly by neglecting all interference terms that the probability for the production of $n$ pairs is

$$
P_{n}=|\langle\overline{0} \mid \Psi\rangle|^{2} \frac{1}{n !} \bar{P}_{1}^{n}
$$

Since the probability for producing no pairs is just $|\langle\overline{0} \mid \Psi\rangle|^{2}$, this formula holds also for $n=0$. By demand- ing that the sum of all probabilities is unity, one finds

$$
|\langle\overline{0} \mid \Psi\rangle|^{2}=\left(\sum_{n=0}^{\infty} \frac{\bar{P}_{1}^{n}}{n !}\right)^{-1}=e^{-\bar{P}_{1}} .
$$

The mean number of created pairs $\bar{n}$ finally becomes

$$
\bar{n}=e^{-\bar{P}_{1}} \sum_{n=0}^{\infty} \frac{n \bar{P}_{1}^{n}}{n !}=\bar{P}_{1}=\sum_{p>0} \sum_{q<0}\left|\bar{S}_{p q}\right|^{2} .
$$

In the next section we will evaluate this number in perturbation theory.

The distribution has the shape of a Poisson distribution

$$
P_{n}=e^{-\bar{n}} \frac{\bar{n}^{n}}{n !}
$$

This is understandable as neglecting interferences corresponds to the independence of creation processes.

\section{B. Perturbation theory}

In perturbation theory the one-particle amplitudes (which are needed only at $t \rightarrow \infty$ and are therefore $S$ matrix elements) are approximated as a series in the electromagnetic coupling constant

$$
a_{n p}=\sum_{i=0}^{\infty} a_{n p}^{(i)} \text {. }
$$

This series is computed using Green's functions satisfying

$$
i \frac{\partial}{\partial t} G\left(t, t^{\prime}\right)=\delta\left(t-t^{\prime}\right)
$$

by the recursive expression

$$
a_{n p}^{(i+1)}=\int d t^{\prime} G_{p}\left(t, t^{\prime}\right) \sum_{p^{\prime}} V_{p p^{\prime}}\left(t^{\prime}\right) a_{n p^{\prime}}^{(i)}\left(t^{\prime}\right)
$$

with the zeroth-order term

$$
a_{n p}^{(0)}=\delta_{n p} .
$$

Since the boundary conditions are given at $t \rightarrow-\infty$, the propagator chosen is the forward propagator

$$
G\left(t, t^{\prime}\right)=-i \Theta\left(t-t^{\prime}\right) .
$$

The reduced one-pair amplitude up to second order is (since $q<0$ and $p>0$ for an electron-positron pair)

$$
\begin{aligned}
\bar{S}_{p q} & =\sum_{n<0}\left(\delta_{n q}+a_{n q}^{(1)}+a_{n q}^{(2)}\right)^{*}\left(\delta_{n p}+a_{n p}^{(1)}+a_{n p}^{(2)}\right) \\
& =a_{q p}^{(1)}+a_{q p}^{(2)}+\sum_{n<0} a_{n q}^{(1) *} a_{n p}^{(1)}+\cdots
\end{aligned}
$$

where the ellipsis represents higher-order terms. The second-order contribution here consists of the true second-order term $a_{q p}^{(2)}$ and of the sum over the product of two first-order terms. Writing explicitly the integrations in these second-order terms

$$
\begin{aligned}
& a_{q p}^{(2)}=(-i)^{2} \int_{-\infty}^{+\infty} d t^{\prime} \int_{-\infty}^{+\infty} d t^{\prime \prime} \sum_{n} V_{p n}\left(t^{\prime}\right) V_{n q}\left(t^{\prime \prime}\right), \\
& \sum_{n<0} a_{n q}^{(1) *} a_{n p}^{(1)}=(-i)(+i) \int_{-\infty}^{+\infty} d t^{\prime} \int_{-\infty}^{+\infty} d t^{\prime \prime} \sum_{n<0} V_{p n}\left(t^{\prime}\right) V_{n q}\left(t^{\prime \prime}\right),
\end{aligned}
$$


one finds for the second-order contribution to pair creation

$$
\bar{S}_{p q}^{(2)}=-\int_{-\infty}^{+\infty} d t^{\prime}\left(\sum_{n>0} \int_{-\infty}^{t^{\prime}} d t^{\prime \prime} V_{p n}\left(t^{\prime}\right) V_{n q}\left(t^{\prime \prime}\right)-\sum_{n<0} \int_{t^{\prime}}^{+\infty} d t^{\prime \prime} V_{p n}\left(t^{\prime}\right) V_{n q}\left(t^{\prime \prime}\right)\right) .
$$

This is exactly the expression one obtains from a perturbation theory with the Feynman propagator

$$
G_{p}^{F}\left(t, t^{\prime}\right)=-i\left\{\begin{array}{l}
\Theta\left(t-t^{\prime}\right) \text { for } p>0 \\
-\Theta\left(t^{\prime}-t\right) \text { for } p<0 .
\end{array}\right.
$$

The Feynman propagator yields the reduced amplitudes $\bar{S}_{p q}$ which are correct if $\langle\overline{0} \mid \Psi\rangle$ is unity. This reflects that the ordinary perturbative vacuum is assumed to be stable.

In the presence of a strong external field the Dirac vacuum becomes unstable. This leads to an additional factor $|\langle\overline{0} \mid \Psi\rangle|^{2}$ in front of the $n$-pair creation probability. While this factor cannot be computed in ordinary perturbation theory, the above reasoning shows that it can be identified - in the approximations made - with

$$
|\langle\overline{0} \mid \Psi\rangle|^{2}=e^{-\bar{n}}
$$

where the average number of pairs $\bar{n}$ is just the reduced single-pair creation probability

$$
\bar{n}=\bar{P}_{1}=\sum_{q<0} \sum_{p>0}\left|\bar{S}_{p q}\right|^{2}
$$

The lowest-order perturbation expansion of $\bar{S}_{p q}$ is

$$
\begin{aligned}
\bar{S}_{p q} & =\sum_{n<0}\left(\delta_{n q}+a_{n q}^{(1) *}\right)\left(\delta_{n p}+a_{n p}^{(1)}\right) \\
& =\sum_{n<0}\left(\delta_{n q}+a_{n q}^{(1) *}\right) a_{n p}^{(1)} \approx a_{q p}^{(1)},
\end{aligned}
$$

since $p>0$, and therefore yields as a lowest-order expression for the average number of created pairs

$$
\bar{n}=\sum_{q<0} \sum_{p>0}\left|\bar{S}_{p q}\right|^{2} \approx \sum_{q<0} \sum_{p>0}\left|a_{q p}^{(1)}\right|^{2} .
$$

The expectation value of the electron number operator $\hat{N}=\Sigma_{p} \hat{N}_{p}$ can be computed without introducing the $|\langle\overline{0} \mid \Psi\rangle|^{2}$ factor, since both $\hat{\vec{d}}_{n}$ and $\hat{\vec{b}}_{n}$ annihilate the time-evolved state $|\Psi\rangle$,

$$
\begin{aligned}
\left\langle\Psi\left|\hat{b}_{p}^{\dagger} \hat{b}_{p}\right| \Psi\right\rangle & =\sum_{n<0} \sum_{m<0} a_{n p}^{*} a_{m p}\left\langle\Psi\left|\hat{\widetilde{d}}_{n} \hat{\tilde{d}}_{m}^{\dagger}\right| \Psi\right\rangle \\
& =\sum_{n<0}\left|a_{n p}\right|^{2},
\end{aligned}
$$

and gives therefore the same expression as for $\bar{n}$,

$$
\langle\Psi|\hat{N}| \Psi\rangle=\sum_{q<0} \sum_{p>0}\left|a_{q p}\right|^{2} .
$$

So the assumption of a Poisson approximation is consistent, and the average number of created pairs is indeed given by summing over the absolute squares of the single-particle scattering matrix.

In the case of pair creation by the electromagnetic fields of heavy ions the $S$-matrix elements $a_{q p}$ of zeroth and first order vanish for $q<0$ and $p>0$. This implies that the intermediate state of the second-order process never lies on the mass shell. The $S$-matrix elements are therefore the same whether the Feynman or the forward propagator is used. The pair-creation amplitude to second order is then from Feynman rules

$$
S_{p q}^{F}=a_{q p}^{(2)}
$$

and the probability to create exactly one pair

$$
P_{1}^{F}=\sum_{p>0} \sum_{q<0}\left|a_{q p}^{(2)}\right|^{2}
$$

This expression is identical to the expression found above for the mean number of pairs created and therefore explicitly violates unitarity, i.e., becomes larger than one. But from this alone it cannot be concluded that the oneparticle amplitudes $a_{q p}^{(2)}$ are wrong; the violation might also be caused by neglecting the factor $\langle\overline{0} \mid \Psi\rangle$. If more than one particle is produced, $P_{1}$ must-for it is to second order identical to $\bar{n}$ - explicitly violate unitarity, even when perturbation theory is still applicable. In this case the unitarity violation stems solely from the neglect of the factor

$$
|\langle\overline{0} \mid \Psi\rangle|^{2}=e^{-\bar{n}}
$$

Therefore, we can use the unitarity violating lowest-order pair creation probability as a lowest-order approximation of the mean number of pairs created; this calculation has been done by several well-known methods, especially by Monte Carlo integration of the Feynman graph [9], by the Weizsäcker-Williams method $[10,1]$, by distortedwave calculations [11], or nonperturbatively in coupledchannel calculations [5], or by direct solutions of the Dirac equation [12]. To get perturbative higher-order results, the $S$-matrix elements $a_{q p}$ of the Dirac equation must be evaluated to higher orders using the forward propagator, which differs from the Feynman propagator in how the integration contour at the mass shell singularity is chosen.

\section{CONCLUSIONS}

We have shown from a general nonperturbative treatment that the pair multiplicity distribution is of Poisson form when interference terms are neglected, and that the average number of pairs can be identified with the (unitarity violating) probability in second-order perturbation theory. The unitarity violation is identified to be not in the perturbative solution of the Dirac equation but in the vacuum-to-vacuum amplitude $\langle 0 \mid \Psi\rangle=\langle 0|S| 0\rangle$, which is neglected in perturbative calculations. Moreover, we have derived expressions that enable us to compute the multiplicity distribution directly when (perturbative or 
nonperturbative) solutions of the Dirac equation are known. Though they involve considerably more integrations than the elementary Feynman graph, it should be possible to compute corrections to the Poisson law numerically when needed.

\section{ACKNOWLEDGMENT}

We would like to thank J. Reinhardt for valuable discussions and for critical reading of the manuscript, and B. Müller for fruitful discussions.
[1] C. A. Bertulani and G. Baur, Phys. Rep. 163, 299 (1988).

[2] T. Lippert, U. Becker, N. Grün, W. Scheid, and G. Soff, Phys. Lett. B 207, 366 (1988); T. Lippert, J. Thiel, N. Grün, and W. Scheid, Int. J. Mod. Phys. A 6, 5249 (1991).

[3] G. Baur, Phys. Rev. D 41, 3535 (1990); Phys. Rev. A 42, 5736 (1990).

[4] M. J. Rhoades-Brown and J. Weneser, Phys. Rev. A 44, 330 (1991).

[5] K. Rumrich, K. Momberger, G. Soff, W. Greiner, N. Grün, and W. Scheid, Phys. Rev. Lett. 66, 2613 (1991); K. Momberger, N. Grün, and W. Scheid, Z. Phys. D 18, 133 (1991).

[6] J. Reinhardt, B. Müller, W. Greiner, and G. Soff, Phys. Rev. Lett. 43, 1307 (1979); G. Soff, J. Reinhardt, B. Müller, and W. Greiner, Z. Phys. A 294, 137 (1980); J. F. Reading and A. L. Ford, Phys. Rev. A 21, 124 (1980).

[7] D. Bailin and A. Love, Introduction to Gauge Field Theory
(Hilger, Bristol, 1986).

[8] C. Itzykson and J.-B. Zuber, Quantum Field Theory (McGraw-Hill, Singapore, 1985).

[9] C. Bottcher and M. R. Strayer, Phys. Rev. D 39, 1330 (1989).

[10] E. Fermi, Z. Phys. 29, 315 (1924); E. J. Williams, Proc. R. Soc. London Ser. A 139, 163 (1933); C. Weizsäcker, Z. Phys. 88, 612 (1934); V. M. Budnev, I. F. Ginzburg, G. V. Meledin, and G. V. Serbo, Phys. Rep. 15, 181 (1975); J. Eichler, ibid. 193, 167 (1990).

[11] U. Becker, N. Grün, and W. Scheid, J. Phys. B 19, 1347 (1986); G. Deco and N. Grün, Phys. Lett. A 143, 387 (1990); F. Decker, Phys. Rev. A 44, 2883 (1991).

[12] C. Bottcher, M. R. Strayer, A. S. Umar, and V. E. Oberacker, Phys. Rev. C 37, 2487 (1988); C. Bottcher, M. R. Strayer, V. E. Oberacker, and A. S. Umar, Phys. Rev. A 41, 1399 (1990). 